\title{
Associative asymmetry in probed recall of serial lists
}

\author{
MICHAEL J. KAHANA and JEREMY B. CAPLAN \\ Brandeis University, Waltham, Massachusetts
}

\begin{abstract}
For pairs of meaningful items (e.g., words), recall accuracy is nearly identical for forward and backward probes. That is, after studying an A-B pair, subjects can recall A given $\mathbf{B}$ as well as they can recall B given A (Kahana, 2002). To assess whether this symmetry property is unique to pairs, we investigated the effects of study direction on probed recall of word triples and serial lists. Two experiments revealed a forward-recalladvantage in both triples and serial lists. In addition, compound cues produced better recall than did single-item adjacent cues, which, in turn, produced better recall than did remote cues. These findings suggest a discontinuity between the associative processes supporting memory for pairs and those supporting memory for sequences of three or more items.
\end{abstract}

Studies of serial order memory have extensively characterized how subjects reproduce sequences of information in order (Crowder \& Greene, 1998; Harcum, 1975; Murdock, 1974). A number of models each provide partial accounts of these data including nearest-neighbor associative chains, position-to-item associations, and hierarchical associative networks (Brown, Preece, \& Hulme, 2000; Burgess \& Hitch, 1999; Lee \& Estes, 1977; Lewandowsky $\&$ Murdock, 1989). Yet, despite over 100 years of research on ordered recall, few, if any, studies have directly examined whether order of study itself influences retrieval efficacy. In contrast, many dozens of studies have examined this question in paired-associate learning, asking whether memory for simple pairs exhibits a forward asymmetry effect (i.e., better forward recall than backward recall). Surprisingly, such asymmetries are exceedingly hard to detect in paired-associate tasks, with many studies producing nearly identicallevels of forward and backward recall (see Ekstrand, 1966, and Kahana, 2002, for reviews of the literature). Although retrieval in paired associate learning is approximately symmetric (with respect to order of study), retrieval in free recall shows marked asymmetries: Forward transitions in recall are significantly more frequent than backward transitions (Howard \& Kahana, 1999; Kahana, 1996; Kahana, Howard, Zaromb, \& Wingfield, 2002). This result is ubiquitous, with the degree of asymmetry

The authors acknowledge support from the National Institutes of Health, Grant MH55687. We are grateful to Arne David Ekstrom for conducting several preliminary studies that led to the development of Experiment 2 and to Kelly Addis for assisting in data collection and for helpful discussions about the analyses of Experiment 2. We also thank Marc Howard, Franklin Zaromb, and Nelson Cowan for their helpful comments on an earlier version of this manuscript. Correspondence concerning this article should be addressed to M. J. Kahana, Volen National Center for Complex Systems, MS 013, Brandeis University, Waltham, MA 02454-0013 (e-mail: kahana@brandeis.edu). being nearly identical for long and short lists (Kahana, 1996), auditory and visual presentation (Kahana, 1996), and for immediate, delayed, and continuous-distractorfree recall (Howard \& Kahana, 1999). Because the free recall task does not impose any constraints on participants' order of report, the asymmetric retrieval in this task could be a consequence of any number of factors, including strategies that tend to favor forward report over backward report. What is not known, however, is whether asymmetry is a more general property of memory for sequences.

Here, we answer two empirical questions. (1) Is there a forward-recall advantage in probed recall of sequences of three or more items? (2) Is there a special advantage, in cuing with two consecutively studied items, for recall of the item preceding or following the cue pair? In what follows, we first review the relevant empirical evidence concerning both of these questions and then report two experiments that demonstrate that, in triples and serial lists, probed recall shows both marked asymmetry and compound cuing effects. These findings have direct implications for models of serial order memory.

In a rare comparison of forward and backward recall of common nouns, Madigan (1971) found that for visually presented lists, forward recall, exhibiting mostly primacy, was not significantly more difficult than backward recall, which exhibited mostly recency. ${ }^{1}$ For auditorally presented lists, however, recall for the last items in the list were greatly enhanced in forward recall, whereas this effect did not reverse to the beginning of the list in backward recall. Li and Lewandowsky $(1993,1995)$ compared the effect of different types of interitem distractors on forward and backward serial recall. They found that whereas some types primarily disrupted forward recall, others primarily disrupted backward recall. These comparisons of forward and backward recall, based on the standard serial recall procedure, confound serial position with order of report, an important determinant of recall performance (e.g., Cowan, Saults, Elliott, \& Moreno, 2002; Dosher \& Ma, 1998). 
An early study by Raskin and Cook (1937) provided more direct evidence for associative asymmetry in serial lists. After their participants learned a list of eight nonsense syllables via the method of anticipation to a criterion of three successive perfect recalls in forward order, they were given a randomly chosen list item and were asked to report the first list item that came to mind (a cross between free association and probed recall). They found that the participants tended to report items near the probe item and exhibited a forward bias in this tendency. However, the method used in this early study cannot determine whether the participants' tendency to produce forward responses reflects an inability to make the transition to the earlier list item or, simply, a preference/bias for making forward responses.

By using the probe technique, one can directly assess participants' ability to recall items, given different cue types (e.g., Murdock, 1968; Posnansky, 1972; Woodward, 1970; Woodward \& Murdock, 1968). In this approach, the experimenter presents a series of items for study. At test, the participants see a cue item and attempt to recall a target that is related to the cue in a specific way (e.g., the instructions may ask the subjects to recall the item that followed the cue in the study list). These probed recall studies found that subjects effectively use both positional cues (e.g., "recall the third word in the list") and sequential cues (e.g., "recall the word that followed RIBBON"). Participants could also use an item as a cue to recall its position in the list (Murdock, 1968). These findings could not select among the classic models of serial learning-positional coding, associative chaining, and hierarchical association .

In relating probed recall of serial lists to serial recall itself, it is important to consider the potential role of compound cuing - that is, the possibility that, during recall, multiple prior items combine to form the cue for the next item. Both probed recall and serial recall studies have yielded evidence for compound cuing. For example, Posnansky (1972) cued subjects for recall of a given target item in three ways: with a position cue, with the prior item as the cue, or with the prior three items as a cue. Cuing with three prior items sometimes enabled recall even when cuing with a single prior item did not. Chance and Kahana (1997) provided further evidence for compound cuing. They examined interresponse times (IRTs) in a task in which participants learned two 15 -word serial lists containing an overlapping series of items. They compared IRTs to recall the item following the overlapping segment across different degrees of overlap-1, 2, 4, or 8 words. A nearestneighbor chaining strategy would be unable to handle overlaps of as little as one item, whereas compound cuing would predict that participants could be resilient to short overlaps. Consistent with the compound cuing hypothesis, critical IRTs were significantly slowed (relative to the other list IRTs) only when the overlapping segment had more than two elements.

\section{EXPERIMENT 1}

In Experiment 1, we analyzed the effects of recall direction and compound cuing in probed recall of triples. We considered triples because we had considerable data on symmetric retrieval in pairs (e.g., Kahana, 2002), and triples are intermediate between pairs and serial lists. We were particularly interested in the possibility that the learning of long serial lists might be seen as participants' stringing together smaller subsequences of items. This is consistent with studies that demonstrate that recall is best when pauses are interposed after every three or four words (e.g., Wickelgren, 1964) and with studies that find scalloped IRT functions, with long pauses after two, three, or four items (e.g., Anderson \& Matessa, 1997; Kahana \& Jacobs, 2000).

\section{Method}

Participants. Seventy-seven undergraduate students participated for either payment or course credit.

Procedure. In each trial, the subjects studied a list of 10 triples, composed of words randomly sampled without replacement from the Toronto Word Pool (Friendly, Franklin, Hoffman, \& Rubin, 1982). For each list, two triples were randomly assigned to each of four repetition conditions (one, two, three, and four presentations). The order of presentation of the triples was randomized, subject to the constraint that all the repetitions were spaced. The two remaining triples were presented last, serving as a recency buffer.

The words in each triple were presented individually, as a series, with each word appearing for $1 \mathrm{sec}$. The first word in each triple appeared on the left side of the screen; as soon as this word was erased, the second word appeared in the center of the screen. After the second word was erased, the third word appeared on the right side of the screen. Although the words appeared in distinct spatial positions, only one word was visible at any moment. A 750-msec interstimulus interval separated the presentation of successive triples. The participants read each word aloud as soon as it appeared on the screen.

During a 3-sec delay between study and test, the computer prompted the subjects to "Get ready to recall." Following this signal, the participants were probed for recall of a missing item in each studied triple. There were six different cue types: two double-item (compound) cues (AB? and ?BC) and four single-item cues (A? , ?B_, _B?, and _?C). We randomly assigned each triple to be tested to one of these six cue types.

The recall cue was always presented visually in an array of three boxes on the screen. For the single-item cues, one of the boxes was empty, one contained a cue item, and the third contained a row of question marks. Vocal recall was recorded digitally and was later scored for accuracy and response time (RT).

During a 1-h session, the subjects performed 18 study-test trials with different lists of triples. This design yielded six responses for each combination of repetition condition and cue type. We separately randomized the selection of items and the order of trials for each subject.

\section{Results and Discussion}

An analysis of variance (ANOVA) revealed significant effects of both cue type $\left[F(5,360)=37.6, M S_{\mathrm{e}}=0.039\right.$, $p<.001]$ and number of presentations $[F(3,216)=240.5$, $\left.M S_{\mathrm{e}}=0.052, p<.001\right]$ on recall probability. Because the interaction between cue type and number of presentations was not statistically significant $[F(15,1080)=1.59$, n.s. $]$, even with a fairly powerful design, we will report comparisons among cue types collapsed across repetition conditions.

Figure 1 shows accuracy and RT for each of the six cue types. Bonferroni-adjusted pairwise comparisons revealed higher recall performance for the forward compound cue (AB?) than for any of the other cue types $(p<.02$ for all 
comparisons). The backward compound cue produced higher recall than all of the single-item cue types $(p<.05$ for all comparisons). Among the single-item cues, adjusted pairwise comparisons revealed a significant forwardbackward asymmetry effect for the A-B pair $(p<.05)$ and a strong trend in favor of asymmetry for the $\mathbf{B}-\mathbf{C}$ pair $(p=.054)$. In summary, forward cues produced better recall than did backward cues, and double-item cues produced better recall than did single-item cues. The asymmetry finding is consistent with (nonsignificant) trends toward asymmetry in a study of triples by Asch and Ebenholtz (1962).

An analysis of correct-response RTs also revealed significant differences among cue types. Correct responses to the forward compound cue were significantly faster than correct responses to all other cue types ( $p \leq .01$ for all pairwise comparisons). Whereas the compound/singleitem cue distinction was dominant for response accuracy, the forward/backward cue distinction was dominant for $\mathrm{RT}$. In particular, the ordering of performance for RT was as follows: RT(AB?) $<$ RT(_B? $)<$ RT $(\mathbf{A}$ ?_) $<$ RT $($ ?BC $)<$ $\mathrm{RT}\left(\_\right.$?C) $<\mathrm{RT}\left(\right.$ ? $\left.\mathbf{B}_{-}\right)$. These results suggest that RT is more sensitive to recall direction, whereas accuracy is more sensitive to compound cuing. Although it is impossible to rule out a speed-accuracy tradeoff here, this is reminiscent of many other dissociations between accuracy and RT in human memory research (see Kahana \& Loftus, 1999, for a review).

\section{Chaining and the Target Ambiguity Problem}

The comparison between probed recall when the cue is the inner item of the triple (B) and when the cue is an outer (terminal) item (A or $\mathbf{C}$ ) is of special interest. Within the framework of associative chaining theory, $\mathbf{A}$ is associated with $\mathbf{B}$, and $\mathbf{B}$ is associated with $\mathbf{C}$. Associations between different triples are either extremely weak or entirely absent. Association between $\mathbf{A}$ and $\mathbf{C}$ is entirely mediated through $\mathbf{B}$. There are no direct $\mathbf{A} \rightarrow \mathbf{C}$ associations in a pure chaining model (Lewandowsky \& Murdock, 1989).

If associations are bidirectional, cuing with $\mathbf{A}$ (for $\mathbf{B}$ ) will activate $\mathbf{B}$. However, cuing with $\mathbf{B}$ (for $\mathbf{C}$ ) will activate both $\mathbf{A}$ and $\mathbf{C}$. How do we focus retrieval on the desired target item, and how do we overcome the associative interference from the nontarget associate? We refer to this as the target ambiguity problem. The problem is not solved simply by assuming asymmetric associations. As long as participants can be cued to recall either the predecessor or the successor of a cue item, the memory system has to resolve the target item in the face of massive associative interference.

We can make this more explicit within the framework of Lewandowsky and Murdock's (1989) application of TODAM to serial recall. In their associative chaining model, a high-dimensionalvector of abstract features represents each list item. As each list item is encoded, the model associates its representation with that of the previous studied item. Both the item representations (vectors) and the associative representations (convolutions of vector pairs) are added to a common memory vector.

Mathematically, the storage process is described by the equation $\mathbf{m}_{j}=\mathbf{m}_{j-1}+\mathbf{f}_{j}+\mathbf{f}_{j} * \mathbf{f}_{j-1}$. In this equation, $\mathbf{f}_{j}$ is a vector representing the $j$ th item in the list, $\mathbf{f}_{j} * \mathbf{f}_{j-1}$ denotes the convolution ${ }^{2}$ of the vectors representing items $j$

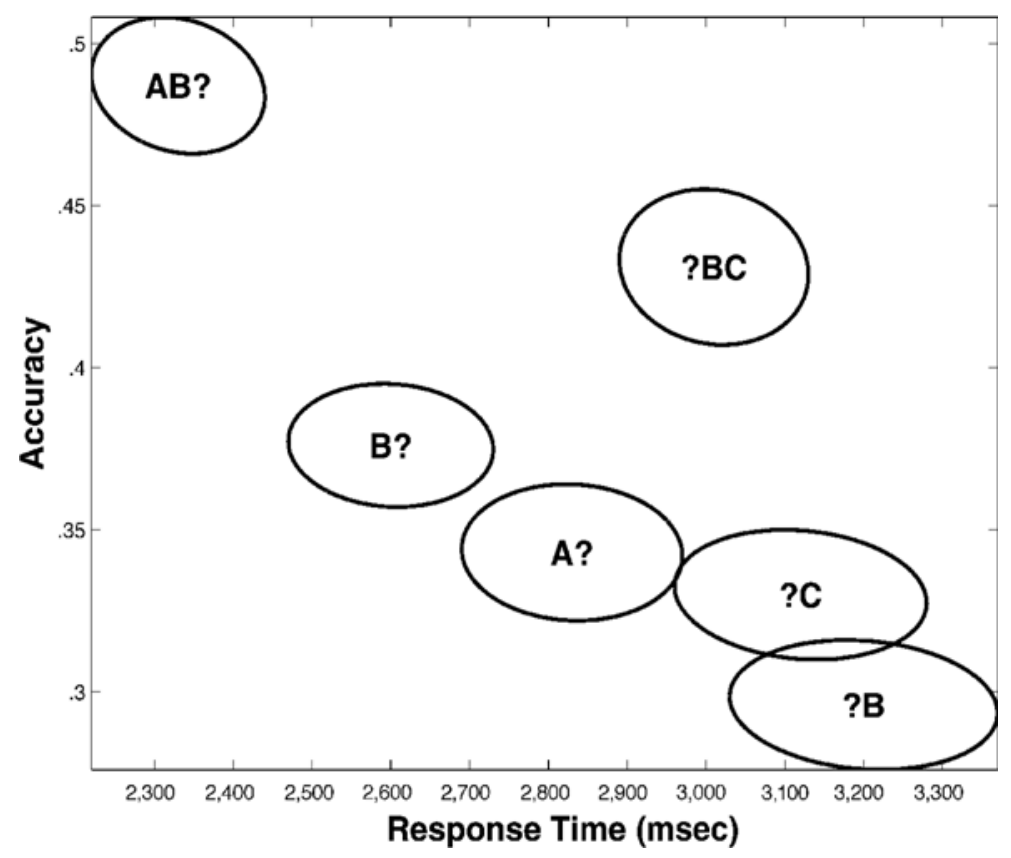

Figure 1. Accuracy versus response time (RT) for probed recall of triples. Cue types are represented by the corresponding symbols, centered around mean accuracy and RT across subjects. Ellipses represent the $95 \%$ confidence region based on a bivariate normal distribution. 
and $j-1$, and $\mathbf{m}_{j}$ is the state of the memory vector after the $j$ th item has been stored. ${ }^{3}$ Once a list with $L$ items has been stored, the memory vector is given by

$$
\mathbf{m}=\mathbf{f}_{1}+\sum_{j=2}^{L} \mathbf{f}_{j}+\mathbf{f}_{j-1} * \mathbf{f}_{j} .
$$

To recall a target item, the cue is correlated with the memory vector (correlation is an approximate inverse of convolution). The probability of recall is a function of the match between the retrieved information, $\mathbf{f}_{x} \# \mathbf{m}$, and the target. In the case of memory for a paired associate, $\mathbf{m}=$ $\mathbf{f}_{1}+\mathbf{f}_{2}+\mathbf{f}_{1} * \mathbf{f}_{2}$, so probing with $\mathbf{f}_{1}$ will unambiguously retrieve $\mathbf{f}_{2}+$ noise, and probing with $\mathbf{f}_{2}$ will unambiguously retrieve $\mathbf{f}_{1}+$ noise.

With lists of three or more items $(L>2)$, retrieval will be unambiguous for probing with the terminal items $\left(\mathbf{f}_{1}\right.$ and $\mathbf{f}_{L}$ ). However, probing with any item from the middle of the list, $\mathbf{f}_{x}$, will retrieve $\mathbf{f}_{x-1}+\mathbf{f}_{x+1}+$ noise. This retrieved information must be deblurred to either $\mathbf{f}_{x-1}$ or $\mathbf{f}_{x+1}$. This poses a target ambiguity problem: The model does not know which item to retrieve. In a winner-take-all network (e.g., Lewandowsky, 1999), the model simply retrieves the strongest representation. However, even in such a network, one must add additional machinery to ensure that an item that is recalled (deblurred) will not be recalled again and again. This is a problem even in determining how associative chaining models might perform serial recall.

Response suppression offers a potential solution for modeling serial recall (e.g., Brown et al., 2000; Burgess \& Hitch, 1999). Within the context of convolution-correlation models, for example, one can keep a running sum of already recalled items in a temporary accumulator vector. The content of this accumulator is subtracted from the retrieved information to obtain an approximate representation of the target item (Murdock, 1993, 1997). However, response suppression does not provide a solution when neither of the two items has already been recalled and the subject is cued to recall one of the two items experimentally, as in our probed recall task.

Simple chaining theory, as outlined above, predicts a substantial performance penalty for cuing with the $\mathbf{B}$ item (relative to cuing with either A or $\mathbf{C}$ ). This target-ambiguity effect should appear even with the inclusion of weaker remote associations between $\mathbf{A}$ and $\mathbf{C}$. This is because cuing with a terminal item, such as $\mathbf{A}$, would retrieve only a weak trace of the remote item, $\mathbf{C}$, whereas cuing with $\mathbf{B}$ would retrieve strong traces of both $\mathbf{A}$ and $\mathbf{C}$.

In the present experiment, we found no significant difference between the ambiguous and the unambiguous forward cues (A?_, B?), or between the ambiguous and unambiguous backward cues (?B_,_?C). There was also no significant difference between the pooled data for the ambiguous and the unambiguous cue types: $P(\mathbf{A}$ ?_) + $P\left(\_\right.$?C $) \approx P\left({ }_{-} \mathbf{B}\right.$ ? $)+P_{-}\left(\mathbf{?} \mathbf{B} \_\right)$. These findings are difficult to reconcile with symmetric chaining models, asymmetric chaining models, or even compound cuing models that allow for weaker remote associations (e.g., Chance \& Kahana, 1997).

\section{EXPERIMENT 2}

In this experiment, we sought to determine whether the asymmetry and compound-cuing results of Experiment 1 generalize to longer lists and in a task in which serial list learning is the participant's primary objective. The participants first mastered a list of words in serial order by the method of repeated study-test trials. After attaining a criterion of one perfect recitation, a short distractor task was performed, and then the participants attempted to recall single words from the study list in response to different cue types. Each participant underwent this process of serial learning, distractor task, and probed recall for 20 lists.

The results of Experiment 1 raised specific questions about probed recall of serial lists. (1) Is there an advantage for compound cues over single-item cues in serial lists? Such a result would be especially important for theories of serial order memory that assume that multiple prior items combine to cue recall of subsequent items (e.g., Chance \& Kahana, 1997; Murdock, 1995a). (2) Is there a forwardrecall advantage with either single- or double-item cues in serial lists? If the forward-recall advantage we found with triples (Experiment 1) is a more general property of serial lists, we would expect to see a similar asymmetry effect in this experiment. We did not examine the effects of target ambiguity in this experiment. This is because only the first and last pairs of each 19-word list could be cued "unambiguously"; for all other cue-target pairs, the subject had to direct retrieval in either the forward or the backward direction, overcoming potential interference from a competing association.

\section{Method}

Participants. Sixty undergraduate and graduate students who were native English speakers participated for payment.

Procedure. The participants took part in five sessions of approximately $1 \mathrm{~h}$ each, with no more than one session per day. The first session was a training session in which the participants first learned a 10 -word list and then a 15 -word list without the distractor and without probed recall. Then the participants learned two 19-word lists, each followed by the distractor task and probed recall. This first session was excluded from analysis. In each of the remaining sessions, the participants learned five 19-word lists, each followed by the distractor task and probed recall. Lists were composed of nouns randomly sampled without replacement from the Toronto Word Pool (Friendly et al., 1982).

During study, the words were presented auditorally at a rate of one word per $1.5 \mathrm{sec}$. At test, the subjects were instructed to vocally recall as much of the list as they could, in order of presentation. Responses were recorded for subsequent scoring of both accuracy and RT. The experimenter remained present throughout all the sessions to determine when the list was recalled perfectly and then to advance the participant to the distractor phase.

The distractor task consisted of equations of the form $A+B+$ $C=D$, where $A, B$, and $C$ were digits from 1 to 9 , displayed on the screen. The equation was either correct (with a probability of .5) or incorrect (off by $+1,-1,+2$, or -2 , each with a probability of .125 ), and the participants were instructed to vocally respond "true" or "false," respectively. Each equation remained on the screen until a response was made. A computerized voice key recorded each response and advanced to the next question. The participants answered 25 randomly chosen questions during each distractor period. 
Following the distractor task, the participants were prompted to "Get ready to recall. ..." The participants were then probed for recall of target items drawn from the serial list. The probes were similar to those used in previous experiments, with two notable differences: (1) Because the only "unambiguous" cues were from the beginning and end of the lists, we focused our analyses on the ambiguous cues and excluded the edge-of-list cues from the analyses, and (2) for completeness, the inner-target cue, $\mathbf{A}$ ?C, and two remote cues, A_? and ?_C, were included. Hence, there were seven types of cues: two single-adjacent cues (A?, ?B), three compound cues (AB?, ?BC, and A?C), and two remote cues: (A_?,?_C). Every list was divided into seven blocks: two pair-blocks and five tripleblocks. A pair-block could be probed only with A? or ?B, whereas a triple-block could be probed only with one of the remaining five cue types. Each cue type appeared with equal probability over the course of the experiment. The list was probed completely, with nonoverlapping cues (i.e., each word was used as a cue item, a target item, or a skipped item, “_," only once), with the cue presentation order randomized. Then the list was once again probed completely with different cue types and a different randomly chosen presentation order, but on the basis of the same blocking scheme. We will report results only from the first complete set of probes. Blocking schemes were chosen randomly for each list. The cue remained on the screen until a response was made. Vocal recall was scored for accuracy and RT, as in Experiment 1.

Artifactual responses (e.g., coughs) made up $1.5 \%$ of all responses (no more than $9.0 \%$ for a given participant) and were excluded from analysis. We also excluded the start and end cue blocks in order to avoid primacy and recency artifacts. ${ }^{4}$

\section{Results and Discussion}

Figure 2 plots accuracy versus RT for each of the seven cue types. To compare the data from this experiment with those from Experiment 1, we performed a $3 \times 2$ ANOVA on cue type (single-adjacent/compound/remote cue types) and cuing direction (forward/backward). Note that at this stage, we excluded the inner cue (A?C) from analysis in order to focus on the two factors, cue type and direction.

For response accuracy, both main effects were significant [cue type, $F(2,118)=76.73, M S_{\mathrm{e}}=0.010, p<.001$; direction, $\left.F(1,59)=45.03, M S_{\mathrm{e}}=0.0075, p<.001\right]$, but the interaction was not significant $[F(2,118)<1]$. Overall, accuracy was highest for compound cues, intermediate for single-adjacent cues, and lowest for remote cues $(p<.001$, Bonferroni-corrected, for all pairwise comparisons). Accuracy for forward cues was also higher than that for backward cues $(p<.001)$. The forward-recall advantage was separately significant for each of the three cue types ( $p<.05$, Bonferroni-corrected), and the compound cuing advantage was significant for both forward and backward cues ( $p<.05$, Bonferroni-corrected).

For correct response RTs, both main effects were statistically significant [cue type, $F(2,118)=114.23, M S_{\mathrm{e}}=$ $2.0 \times 10^{6} \mathrm{msec}^{2}, p<.001$; direction, $F(1,59)=57.80$, $\left.M S_{\mathrm{e}}=1.2 \times 10^{6} \mathrm{msec}^{2}, p<.001\right]$. In addition to these main effects, the interaction between direction and cue type was statistically significant $\left[F(2,118)=6.03, M S_{\mathrm{e}}=\right.$ $\left.1.3 \times 10^{6} \mathrm{msec}^{2}, p<.01\right]$. Post hoc comparisons revealed that the single-adjacent and compound cues were each performed more quickly than remote cues $(p<.001$, Bonferroni-corrected) but the compound and singleadjacent cues did not differ significantly in RT. Overall,

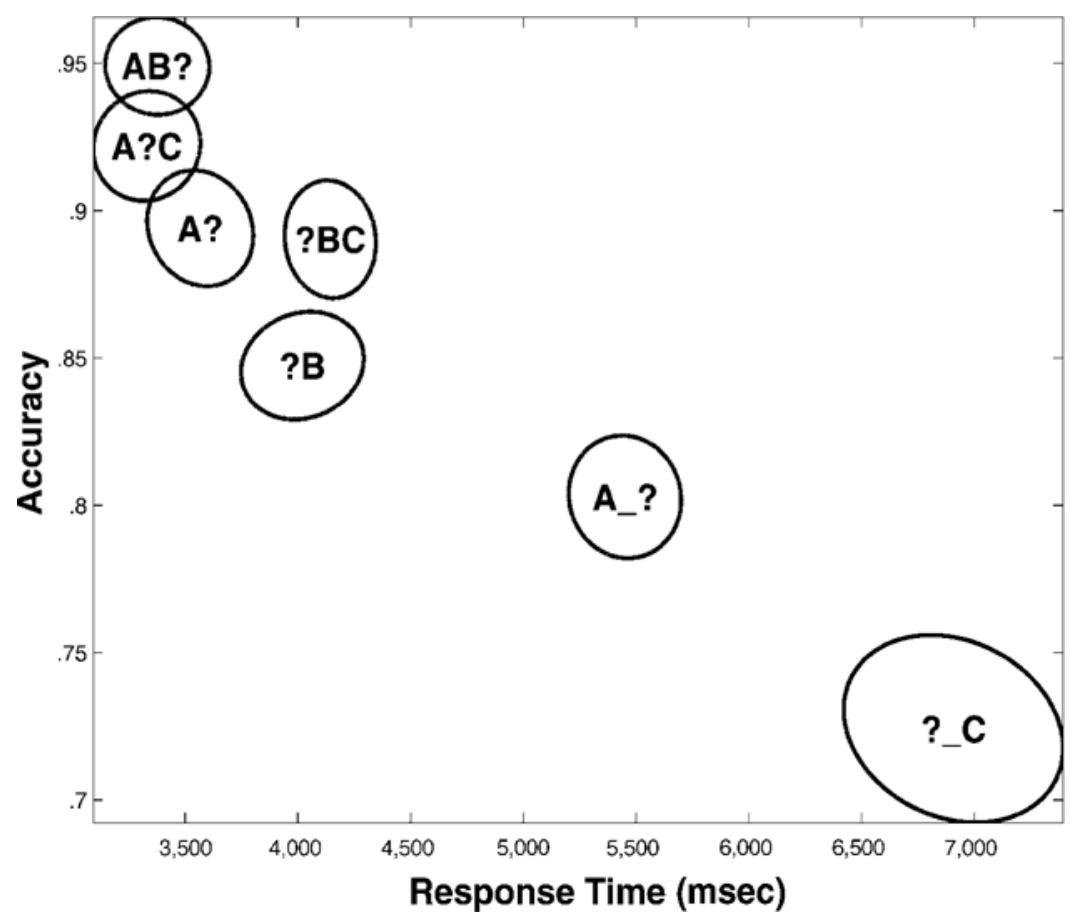

Figure 2. Accuracy versus response time (RT) for probed recall of serial lists. Cue types are represented by the corresponding symbols, centered around mean accuracy and RT across subjects. Ellipses represent the $95 \%$ confidence region based on a bivariate normal distribution. 
correct responses were faster to forward cues than to backward cues. Bonferroni-corrected paired-samples $t$ tests showed that the forward-recall advantage was separately significant for each of the three cue types $(p<.01)$. However, the advantage for compound cuing held only for the forward direction $(p<.001)$, and not for the backward direction, reflecting the interaction effect.

These results generalize the primary findings of Experiment 1 . In serial lists, significant benefits accrue from forward cuing and compound cuing, with these two factors combining to provide the best performance for the forward compound cue.

Asymmetry with compound cues appeared in both accuracy and latency. This effect reinforces the view that asymmetry is a basic property of retrieval from multi-item lists (cf. Howard \& Kahana, 1999; Kahana, 1996). With singleitem cues, asymmetry effects tended to be smaller, although they appeared consistently in both of our experiments.

If associations formed in serial learning are asymmetric, as is consistent with our findings thus far, then when subjects fail to recall a target item, the forward bias might show up in their intrusion patterns. Figure 3 plots, for forward and backward single-adjacent cues (A? and ?B), the proportion of within-list intrusions at each lag relative to the target item. For each cue type, only the participants who made at least one within-list intrusion were included in the within-list analyses. The plots plainly show an asymmetry in intrusions; the participants were more likely to erroneously recall items following the cue block than items pre- ceding it. Paired-samples $t$ tests show that the difference between the rate of intrusions for the items immediately following the probe and the items immediately preceding the probe was significant for the backward probe and shows a trend toward significance for the forward probe [A?, $t(36)=1.90, p=.065 ; \mathbf{~ ? B}, t(48)=2.26, p<.05]$. For the participants included in either cue type analysis, this forward bias was significantly greater for backward than for forward cuing direction $[t(56)=-2.27, p<.05]$.

Our finding of a forward compound cuing advantage is consistent with hierarchical associative models. These models posit that within a triple, $\mathbf{C}$ is associated with a node representing AB. Although such models might also be able to account for the advantage of the backward compound cue, $p(\mathbf{B C} \rightarrow \mathbf{A})>p(\mathbf{B} \rightarrow \mathbf{A})$, this prediction would be highly sensitive to the specific implementation. Chaining models cannot account for the compound cuing results without incorporating some version of remote association (e.g., Murdock, 1995b).

\section{GENERAL DISCUSSION}

In this paper, we asked whether similar associative processes underlie memory for pairs and memory for sequences. Associative symmetry is a basic property of memory for symbolic pairs learned episodically (Asch \& Ebenholtz, 1962; Mandler, Rabinowitz, \& Simon, 1981; Murdock, 1962, 1965, 1966). In a recent analysis of this literature, Kahana (2002) noted that empirical violations

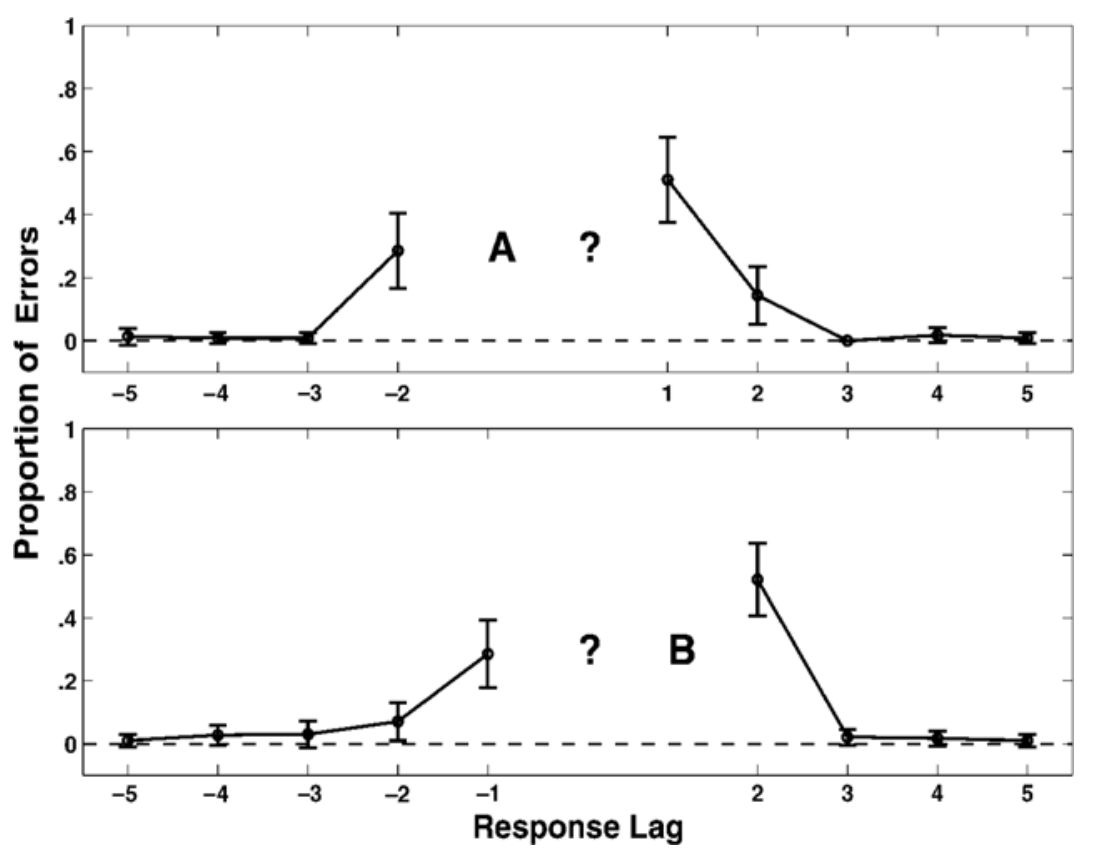

Figure 3. Within-list intrusions. The proportion of within-list intrusions at each lag are plotted for the forward (A?) and backward (?B) single-adjacent cue types, respectively. Lag is relative to the target item (?), so that the target item has lag $=0$. Only participants who made at least 1 within-list intrusion on a given cue type were included (37 participants for $A$ ?, 49 participants for ?B). The total number of within-list intrusions was 67 for $A$ ? and 98 for ?B. Error bars denote $95 \%$ confidence intervals. 
of the symmetry principle are rare. We wondered whether forward and backward retrieval in longer lists also obeyed the symmetry principle. Although previous studies suggested that forward and backward serial recall are differentially influenced by various manipulations (e.g., Li \& Lewandowsky, 1993, 1995; Madigan, 1971), direct comparisons of forward and backward probed recall had not been made. By varying the types of cues given in the probed recall task, we examined the effects of recall direction, compound cuing, and target ambiguity in triples (Experiment 1) and in fully learned serial lists (Experiment 2).

Probes of forward versus backward recall in triples and serial lists demonstrate a clear breakdown in the principle of associative symmetry, with forward recall being superior to backward recall on both accuracy and latency measures. In Experiment 1, in which participants studied word trigrams, forward recall was both more accurate and faster than backward recall for single- and for double-item cues. Experiment 2 served a critical role in directly linking the analysis of recall direction and compound cuing with serial learning. After a 19-word list was mastered, subsequent probed recall revealed significant effects of compound cuing and of asymmetry for single-item adjacent, single-item remote, and compound cues. The participants also exhibited asymmetry in their pattern of within-list intrusions. Specifically, they were more likely to mistakenly recall items in the forward direction, even when cued for backward recall (see Figure 3). One interpretation of this pattern is that when subjects fail to retrieve the correct item (perhaps by their own assessment), they resort to a backup strategy-something akin to free recalling from the list to produce candidate items. This could account for the adjacency and asymmetry in Figure 3, which are also found in the conditional response probability curves for free recall (Howard \& Kahana, 1999; Kahana, 1996; Kahana et al., 2002), as well as for the cued free recall in Raskin and Cook (1937).

The forward-recall advantage in probed recall of triples and serial lists, coupled with the symmetric recall of pairs (Kahana, 2002; Rizzuto \& Kahana, 2001), presents a new puzzle for theories of associative memory. Some memory models assume symmetric associative operations (Hintzman, 1986; Metcalfe, 1985; Metcalfe-Eich, 1982; Murdock, 1982,1997), whereas others allow for separate weights on forward and backward associations (Gillund \& Shiffrin, 1984; Humphreys, Bain, \& Pike, 1989; Humphreys, Pike, Bain, \& Tehan, 1989; Kahana, 1996; Raaijmakers \& Shiffrin, 1980). If these associative models are to apply to both paired-associate and serial learning, some additional assumptions are required. One possibility is that associations are inherently asymmetric but that symmetry in pairs arises from some other process. For example, in the SAM model (Gillund \& Shiffrin, 1984), one can allow for asymmetric changes in associative strength, with stronger forward than backward associations (Gillund \& Shiffrin, 1984; Kahana, 1996). We can further assume that rehearsal causes items to cycle through the rehearsal buffer (shortterm store, STS) in presentation order. In free and serial recall, asymmetries will appear owing to the asymmetric associative strengths. In paired-associate learning, however, because the two members of a pair occupy STS alone (Raaijmakers \& Shiffrin, 1981), the second member of the pair becomes associated in the forward direction with the first member of the pair each time the pair repeats (A-B-A-B-A . . .), thereby attenuating the underlying asymmetry. An early study by Raskin and Cook (1937) suggests that some form of last-to-first rehearsal takes place in associative learning, as evidenced by a tendency for subjects' recalls to wrap around the list boundaries.

The asymmetry seen in triples and serial lists is especially pronounced when retrieval is cued with two successive items. Consistent with the special status of the forward compound cue, Experiments 1 and 2 showed that probing with forward compound cues consistently produces better recall performance than does probing with forward single-item cues. It is possible that the advantage of the compound cue derives from its similarity to how participantslearn serial lists. The "effective" cue in serial learning may be more appropriately thought of as a run of successive items, rather than as a single-adjacent item. This is consistent with models that assume that multiple prior items combine to cue subsequent recalls (e.g., Chance \& Kahana, 1997; Murdock, 1995a).

In Experiment 1, we examined a basic prediction of associative chaining models of serial recall. If a word trigram (denoted $\mathbf{A B C}$ ) is coded as two associations, $\mathbf{A}-\mathbf{B}$ and $\mathbf{B}-\mathbf{C}$, cuing with $\mathbf{A}$ will activate $\mathbf{B}$, whereas cuing with $\mathbf{B}$ will activate both $\mathbf{A}$ and $\mathbf{C}$. This presents a challenge to the models: How can participants focus retrieval on the desired target item, and how can they overcome the associative interference from the nontarget associate? To be able to selectively recall either the predecessor or the successor of a cue item, the memory system has to overcome considerable associative interference. Contrary to our expectations and to the predictions of associative chaining theory, Experiment 1 failed to show a significant effect of target ambiguity on recall performance.

This failure to find associative interference in serial lists is not restricted to our case of probed recall of word triples. For example, after studying series of items containing a single (spaced) repeated item, participants show impaired recall for the second instance of the repeated item. According to an associative framework, participants should have trouble remembering the items following the two repetitions. Contrary to this prediction, participants are not impaired at recalling the items following the repeated item but are both less accurate (Crowder, 1968) at recalling the second repeated item itself and slower (Kahana \& Jacobs, 2000). Similarly, Primoff (1938) found that double-function lists (chains of paired associates of the form $\mathbf{A}-\mathbf{B}, \mathbf{B}-\mathbf{C}, \mathbf{C}-\mathbf{D}, \ldots$. .) were much more difficult to learn than serial lists (A-B-C-D-E . . .). This difficulty was later shown to be largely due to the added associative interference of the backward association found in the paired-associate lists (Slamecka, 1976). It seems that the organization of serial lists enables participants to overcome the associative interference that plagues the acquisition of double-function lists. 
Failure to observe associative interference in serial lists could be taken as support for models that do not rely on associative processes in serial recall. Indeed, a new generation of models of serial order memory are based on sophisticated notions of positional coding (Brown et al., 2000; Burgess \& Hitch, 1999). These models, although applied extensively to serial recall, have not yet been applied to probed recall. Nonetheless, one could imagine how they might account for probed recall and could test them against our empirical results.

We first assume that presentation of the probe recovers some representation of its serial position. This positional code might then be shifted, either forward or backward, to the desired relative serial position of the target. This shifted positional code would then serve as a retrieval cue for the target item. One could assume that this operation is symmetric, or one could assume that shifting is more readily accomplished in, say, the forward direction. This latter assumption could be used to explain the forwardrecall advantage we observe in probed recall.

If we assume that each item can retrieve its positional code, having the code for the nearest neighbor should suffice, and adding the positional code from a more distant item (as in the case of compound cuing) should not facilitate recall. To explain compound cuing effects within positional-coding models, one has to assume that a probe item does not necessarily succeed in recovering its positional context. In this case, having additional context cues should help performance, as we have observed. Alternatively, if we suppose that the shifting operation is fallible, displaying extra cue items could serve to rule out possible positional intrusions.

To evaluate this possibility, consider Figure 3 . The bulk of the within-list intrusions come from serial positions adjacent to the cue block. Adding an additional adjacent item to the cue (i.e., $\mathbf{A B}$ ? instead of $\mathbf{B}$ ? or $\mathbf{P B C}$ instead of ?B) should prevent participants from making some withinlist intrusions, which could account for the advantage of compound cuing in both forward and backward recall (Figure 2). On the other hand, by this reasoning, one would predict that the inner cue (A?C) should outperform the forward compound cue. This is clear if one starts with the single-adjacent forward cue (A?) and then computes the proportion of within-list intrusions that are ruled out by presenting the additional probe item. In the case of $\mathbf{A B}$ ?, we eliminate the intrusions that are due to lag $=-2$, or roughly $30 \%$ of the intrusions. Presenting A ?C should eliminate the within-list intrusions at lag $=+1$, or roughly $50 \%$ of the intrusions. Therefore, if differences in performance were exclusively due to the effects of blocking candidates for within-list intrusions, we would predict that $\mathbf{A}$ ?C should show higher accuracy than $\mathbf{A B}$ ?, but the opposite is the case (Figure 2).

\section{CONCLUSIONS}

We have shown a clear forward-recall advantage for both single and compound cues, in triples as well as in serial lists. This retrieval asymmetry stands in contrast to the symmetric recall seen regularly in paired associates. That is, after studying an A-B pair, subjects can recall $\mathbf{A}$ given $\mathbf{B}$ as accurately as they can recall $\mathbf{B}$ given $\mathbf{A}$ (Kahana, 2002; Rizzuto \& Kahana, 2001). These findings suggest a discontinuity between the associative processes supporting memory for pairs and those supporting memory for sequences of three or more items. This discontinuity poses a challenge to any memory model that uses associative mechanisms to explain serial order phenomena, whether by associating items with each other, with their ordinal list positions, or in a hierarchical structure.

\section{REFERENCES}

Anderson, J. R., \& Matessa, M. (1997). A production system theory of serial memory. Psychological Review, 104, 728-748.

Asch, S. E., \& EBEnHoltz, S. M. (1962). The principle of associative symmetry. Proceedings of the American Philosophical Society, 106, 135-163.

Brown, G. D. A., Preece, T., \& Hulme, C. (2000). Oscillator-based memory for serial order. Psychological Review, 107, 127-181.

Burgess, N., \& Hitch, G. J. (1999). Memory for serial order: A network model of the phonologicalloop and its timing. Psychological Review, 106, 551-581.

Chance, F. S., \& Kahana, M. J. (1997). Testing the role of associative interference and compound cues in sequence memory. In J. Bower (Ed.), Computationalneuroscience: Trends in research (pp. 599-603). New York: Plenum.

Cowan, N., Saults, J. S., Elliott, E. M., \& Moreno, M. (2002). Deconfounding serial recall. Journal of Memory \& Language, 46, 153177.

Crowder, R. G. (1968). Evidence for the chaining hypothesis of serial verbal learning. Journal of Experimental Psychology, 76, 497-500.

Crowder, R. G., \& Greene, R. L. (1998). Serial learning: Cognition and behavior. In E. Tulving \& F. I. M. Craik (Eds.), The Oxford handbook of memory (pp. 125-135). Oxford: Oxford University Press.

Dosher, B. A., \& MA, J. J. (1998). Output loss or rehearsal loop? Outputtime versus pronunciation-timelimits in immediate recall for forgettingmatched materials. Journal of Experimental Psychology: Learning, Memory, \& Cognition, 24, 316-335.

Ekstrand, B. R. (1966). Backward associations. Psychological Bulletin, 65, 50-64.

Friendly, M., Franklin, P. E., Hoffman, D., \& Rubin, D. C. (1982). The Toronto Word Pool: Norms for imagery, concreteness, orthographic variables, and grammatical usage for 1,080 words. Behavior Research Methods \& Instrumentation, 14, 375-399.

Gillund, G., \& Shiffrin, R. M. (1984). A retrieval model for both recognition and recall. Psychological Review, 91, 1-67.

Harcum, E R. (1975). Serial learning and paralearning: Controlprocesses in serial acquisition. New York: Wiley.

Hintzman, D. (1986). "Schema abstraction" in a multiple-trace memory model. Psychological Review, 93, 411-428.

Howard, M. W., \& KaHANA, M. J. (1999). Contextual variability and serial position effects in free recall. Journal of Experimental Psychology: Learning, Memory, \& Cognition, 25, 923-941.

Humphreys, M. S., Bain, J. D., \& Pike, R (1989). Different ways to cue a coherent memory system: A theory for episodic, semantic, and procedural tasks. Psychological Review, 96, 208-233.

Humphreys, M. S., Pike, R., Bain, J. D., \& Tehan, G. (1989). Global matching: A comparison of the SAM, Minerva II, Matrix, and TODAM models. Journal of Mathematical Psychology, 33, 36-67.

KaHANA, M. J. (1996). Associative retrieval processes in free recall. Memory \& Cognition, 24, 103-109.

Kahana, M. J. (2002). Associative symmetry and memory theory. Memory \& Cognition, 30, 823-840.

Kahana, M. J., Howard, M. W., Zaromb, F., \& Wingfield, A. (2002). Age dissociates recency and lag-recency effects in free recall. Journal of Experimental Psychology: Learning, Memory, \& Cognition, 28, 530-540. 
KahanA, M. J., \& JACOBS, J. (2000). Inter-response times in serial recall: Effects of intraserial repetition. Journal of Experimental Psychology: Learning, Memory \& Cognition, 26, 1188-1197.

Kahana, M. J., \& Loftus, G. (1999). Response time versus accuracy in human memory. In R. J. Sternberg (Ed.), The nature of cognition (pp. 322-384). Cambridge, MA: MIT Press.

LeE, C. L., \& EsTES, W. K. (1977). Order and position in primary memory for letter strings. Journal of Verbal Learning \& Verbal Behavior, 16, 395-418.

LEWANDOWSKY, S. (1999). Redintegration and response suppression in serial recall: A dynamic network model. International Journal of Psychology, 34, 434-446.

Lewandowsky, S., \& Murdock, B. B. (1989). Memory for serial order. Psychological Review, 96, 25-57.

LI, S., \& LEWANDOWSKY, S. (1993). Intralist distractors and recall direction: Constraints on models of memory for serial order. Journal of Experimental Psychology: Learning, Memory, \& Cognition, 19, 895908.

LI, S., \& LewANDowsKy, S. (1995). Forward and backward recall: Different retrieval processes. Journal of Experimental Psychology: Learning, Memory, \& Cognition, 21, 837-847.

Madigan, S. (1971). Modality and recall order interactions in shortterm memory for serial order. Journal of Experimental Psychology, 87, 294-296.

Mandler, G., Rabinowitz, J. C., \& Simon, R. A. (1981). Coordinate organization: The holistic representation of word pairs. American Journal of Psychology, 92, 209-222.

Metcalfe, J. (1985). Levels of processing, encoding specificity, elaboration, and CHARM. Psychological Review, 92, 1-38.

Metcalfe-Eich, J. (1982). A composite holographic associative recall model. Psychological Review, 89, 627-661.

MuRdock, B. B. (1962). Direction of recall in short term memory. Journal of Verbal Learning \& Verbal Behavior, 1, 119-124.

Murdock, B. B. (1965). Associative symmetry and dichotic presentation. Journal of Verbal Learning \& Verbal Behavior, 4, 222-226.

Murdock, B. B. (1966). Forward and backward associations in paired associates. Journal of Experimental Psychology, 71, 732-737.

Murdock, B. B. (1968). Serial order effects in short-term memory. Journal of Experimental Psychology Monographs, 76(4, Pt. 2), 1-15.

MuRdock, B. B. (1974). Human memory: Theory and data. Potomac, MD: Erlbaum.

Murdock, B. B. (1982). A theory for the storage and retrieval of item and associative information. Psychological Review, 89, 609-626.

Murdock, B. B. (1993). TODAM2: A model for the storage and retrieval of item, associative, and serial-order information. Psychological Review, 100, 183-203.

Murdock, B. B. (1995a). Developing TODAM: Three models for serialorder information. Memory \& Cognition, 23, 631-645.

Murdock, B. B. (1995b). Similarity in a distributed memory model. Journal of Mathematical Psychology, 39, 251-264.

Murdock, B. B. (1997). Context and mediators in a theory of distributed associative memory (TODAM2). Psychological Review, 104, 839-862.

Posnansky, C. J. (1972). Probing for the functional stimuli in serial learning. Journal of Experimental Psychology, 96, 184-193.

PRIMOFF, E. (1938). Backward and forward associations as an organizing act in serial and in paired-associate learning. Journal of Psychology, 5, 375-395.

RAaiJMAKers, J. G. W., \& Shiffrin, R. M. (1980). SAM: A theory of probabilistic search of associative memory. In G. H. Bower (Ed.), The psychology oflearning and motivation: Advances in research and theory (Vol. 14, pp. 207-262). New York: Academic Press.

RaAijmakers, J. G. W., \& Shiffrin, R. M. (1981). Order effects in recall. In J. [B.] Long \& A. [D.] Baddeley (Eds.), Attention and performance IX (pp. 403-415). Hillsdale, NJ: Erlbaum.

RAskin, E., \& CooK, S. W. (1937). The strength and direction of associations formed in the learning of nonsense syllables. Journal of Experimental Psychology, 20, 381-395.

Rizzuto, D. S., \& Kahana, M. J. (2001). An autoassociative model of paired associate learning. Neural Computation, 13, 2075-2092.

SlamecKa, N. J. (1976). An analysis of double-function lists. Memory \& Cognition, 4, 581-585.

WiCKELGREN, W. A. (1964). Size of rehearsal group and short-term memory. Journal of Experimental Psychology, 68, 413-419.

WoodWARD, A. E. (1970). Continuity between serial memory and serial learning. Journal of Experimental Psychology, 85, 90-94.

Woodward, A. [E.], \& Murdock, B. B. (1968). Positional and sequential probes in serial learning. Canadian Journal of Psychology, 22, 131-138.

\section{NOTES}

1. This finding may appear surprising, because studies of memory span for digits usually exhibit a substantial advantage for forward recall. This asymmetry effect in digit recall is likely to be a confound of the nature of the materials. Unlike words, sequences of digits are quickly and easily recoded into unitary representations (i.e., the sequence 5-3-9-2$8-1$ can be stored as two units, 539 followed by 281 ). This unitization of subsequences of digits would generally impair backward recall.

2. If each of two item vectors has $N$ elements, the convolution will be a vector with $2 N-1$ elements. The convolution of two vectors, $\mathbf{f}$ and $\mathbf{g}$, is defined by the equation $(\boldsymbol{f} * \boldsymbol{g})_{m}=\sum_{i} f_{i} g_{m-i}$, where $m$ is the index to the elements in the convolution vector and $\mathrm{i}$ indexes the elements in the item vectors $\boldsymbol{f}$ and $\mathbf{g}$. The asterisk (*) denotes the convolution operator. Correlation is an approximate inverse of the convolution operation. $f$ correlated with $\mathbf{g}$ is defined by the equation $(f \# g)_{m}=\sum_{i} f_{i} g_{m+i}$, where the pound sign (\#) denotes the correlation operator.

3. In writing the storage equation for the Lewandowsky and Murdock (1989) model, we are omitting scaling parameters that have the effect of varying the allocation of attention from associative encoding at early list positions to item encoding at later positions.

4. We conducted a a cue block $(7) \times$ cue type $(7)$ ANOVA to assess the effect of cue block on differences among cue types. For recall accuracy, there was a significant main effect of cue block $[F(6,354)=2.56$, $\left.M S_{\mathrm{e}}=0.0051, p<.05\right]$ and of cue type $\left[F(6,354)=32.58, M S_{\mathrm{e}}=\right.$ $0.0059, p<.001]$ but no significant interaction $[F(36,2124)=0.58$, n.s.]. Examination of the Bonferroni-adjusted pairwise comparisons for cue block revealed significant differences $(p<.05)$ only between Block 4 and Blocks 1 and 3. For RT, there was a significant main effect of cue block $\left[F(6,354)=2.50, M S_{\mathrm{e}}=9.0 \times 10^{6} \mathrm{msec}^{2}, p<.05\right.$, and of cue type $\left[F(6,354)=39.06, M S_{\mathrm{e}}=9.8 \times 10^{6} \mathrm{msec}^{2}, p<.001\right]$ and no significant cue type $\times$ cue block interaction $[F(36,2124)=0.86$, n.s.]. None of the post hoc comparisons among blocks was significant to $p<$ .05 , Bonferroni corrected. We excluded the terminal blocks from the subsequent analyses.

(Manuscript received January 12, 2000; revision accepted for publication July 3, 2001.) 